\title{
Modeling Economic Activities and Random Catastrophic Failures of Financial Networks via Gibbs Random Fields
}

\author{
Levent Onural $^{1}$ (D) $\cdot$ Mustafa Çelebi Pınar ${ }^{2} \cdot$ Can Fırtına $^{3}$ \\ Accepted: 9 July 2020 \\ ๑) Springer Science+Business Media, LLC, part of Springer Nature 2020
}

\begin{abstract}
The complicated economic behavior of entities in a population can be modeled as a Gibbs random field (GRF). Even with simple GRF models, which restrict direct statistical interactions with a small number of neighbors of an entity, real life economic and financial activities may be effectively described. A computer simulator is developed to run empirical experiments to assess different coupling structures and parameters of the presented model; it is possible to test many economic and financial models and policies in terms of their transient and steady-state consequences.
\end{abstract}

Keywords Economic networks · Financial networks · Gibbs random fields · Markov random fields $\cdot$ Metropolis algorithm

\section{Introduction}

There is no doubt that the global network of economic activities is complicated. Individual decisions and actions at various levels combine at macro levels and thus determine the global activity patterns. Ever since the field of economics emerged there has been a debate over the mechanisms and drivers of economic interactions among different agents composing the economy as a whole and its impact on equilibrium aspects such as prices and interest rates.

Two different conceptual views are present in the fields of macroeconomics and microeconomics. While macroeconomics is predominantly focused on the behavior of complete economies reacting to external stimuli, microeconomics is concentrated on the behavior of the smallest components of an economy, namely the various

\footnotetext{
$凶 \quad$ Levent Onural

onural@bilkent.edu.tr

1 Department of Electrical and Electronics Engineering, Bilkent University, 06800 Ankara, Turkey

2 Department of Industrial Engineering, Bilkent University, 06800 Ankara, Turkey

3 Department of Computer Engineering, Bilkent University, 06800 Ankara, Turkey
} 
actors contained within it, interacting to form an aggregate of economic behavior. The macroeconomic view sees the processes of economic behavior as being relatively static, with the belief that these processes inherently lead to an equilibrium state. A change in the economic environment will alter this equilibrium, once the change has concluded, the economy will return to another equilibrium position. Specific relationships between behavior and outcomes at a granular level may be ignored. The microeconomic view, in contrast, places utmost importance on individuals and their decision making, the core belief being that human behavior is not always rational and processes inherent in the working economy fluctuate depending on individual or firm behavior, and cannot therefore be considered in isolation from the behavior of other economic agents.

The work presented here focuses on the results of the interaction of a set of economic agents, be it individuals, households or banks (or other institutions). One can certainly not ignore the probabilistic nature of economic activities. Even though it is clearly feasible to define and run even a single entity economy, almost all economic activities are social, and therefore, involve a large scale of interactions among many economic entities. Interactions among the entities (players) and the time evolution of all such interactions, inputs and outputs constitute a complicated process.

Understanding the inner workings of such a complicated probabilistic system is surely interesting from an academic point of view. Furthermore, such an understanding will then surely lead to various policy actions both at the micro and macro levels to control this complicated random system; such policies can be successful only if the underlying mechanisms are well understood.

Central to the our work is the dynamic interplay between economic entities (agents) operating in a highly competitive environment. From this perspective our contribution can be placed within the literature of evolutionary economics. The evolutionary economics concept holds that the economy can be perceived as a large population of diverse agents, incorporating e.g., technology changes and self-transformations of industry structures or changing production or consumption patterns of a group of entities (Nelson et al. 2018). Empirical studies with this population perspective are feasible, and interactive behavior within the population can theoretically be modeled. With the availability of modern computational tools, much of the focus in this area has moved toward formal simulation modeling, where multiple combinations of diverse economic elements can be synthesized and overall economic behavior and equilibrium outcomes examined under varying environmental conditions.

The focus of the present paper is to provide a mathematical model that represents the effects of dynamic interactions between various agents of an economic system. In particular, we highlight the relationships between aggregate macroeconomic effects of the diverse microeconomic actions of individual entities. Based on the presented model, a simulation tool that enables one to observe and analyze the behavior of economic entities under different economic environments, particularly in situations of economic or financial distress, is developed.

The approach undertaken was to establish a theoretical model suitable for a range of interactions among a potentially large number of entities, in a simulation context without the constraining limitations of analytical models which necessarily require simplifying assumptions that detract seriously from reality (Acemoglu et al. 2015). 
The goals of this study are to (i) provide a general and flexible hierarchical random field model to represent economic behavior of a large and complicated system that consists of individual entities, (ii) provide a simulation algorithm based on the provided model, and implement it as a software tool to track the time evolution of the model, (iii) show that actual set of parameters that successfully model such a complicated random system could indeed be quite small, (iv) provide a list of associations between the model structure together with their parameters, and the resultant transient and steadystate behavior of the macro economic system, (v) use the developed model and the simulator to make similar assessments, associations and statements on various related side issues within the broader system; one such example is the reduced system of financial networks, flow of funds as a consequence of loan activities, and the robustness (or the vulnerability) of such networks to random catastrophic failures that may hit some entities and then propagate to the network.

We define an economic entity as a unit which is capable of producing, consuming and storing (accumulating) economic wealth. Surely, any or all of these three key activities can be further divided to achieve a more detailed model and analysis. However, it may be desirable to consolidate various activities into these three main economic behaviors, for each entity. Simpler models are mathematically easier to handle and related simulations are easier to implement.

The simulation model developed here has a firm theoretical grounding as detailed in the rest of the paper.

The Gibbs random fields, or equivalently, Markov random fields are powerful tools to model interactions in spatial systems. The model fully derives the macro behaviors and patterns in larger scales starting from simple micro level interactions among individual players and their small neighborhoods. For example, the macro scale form of a forest can be found from simple forms of interactions with a single tree with its small number of neighbors. Similarly, group behavior of insects, birds, etc., can be found from simple forms of simple interactions of one entity with its few neighbors. The propositions here in this work are, (i) The behavior of an entity in an economic system is strongly affected by the behaviors of peers, social media connections, friends, families, etc., of that entity; we collectively call those directly linked surroundings of an entity as its "neighbors". (ii) The nature of that small scale and easy to understand interactions between a single entity and its neighbors, will then impose predictable and computable macro level economic patterns. It is rather easy to analyze and model those simple interactions between an entity and its neighbors. (iii) Through the GRF (or equivalently the MRF) model, those simple interactions outlined in (i) and (ii) lead to the macro level global behavior patterns; macroeconomics deals with such patterns. (iv) Since local interactions lead to global patterns, and since the nature of those global patterns are tightly linked to those local interactions via th GRF model, a policy maker who desires a specified global state and pattern, may achieve such goals maybe by working on finding ways to alter those simple entity-neighbor relations which will then create the desired global state.

The versatility of our model and simulation tool makes it widely applicable to the solution of varied economic or financial problems.

The adaptation of a given system structure by altering its parameters to fit an observed random process, is well studied in the engineering literature (see, for exam- 
ple, Haykin 1986 or its later editions). Gibbs random field (or equivalently Markov random field) parameter estimation problem is also studied (Gurelli and Onural 1994). Estimation of the parameters of the proposed model to fit a specific empirical data, or better yet, adaptation of such parameters over time, will be a natural extension of the study presented in this paper. This will also address the Lucas' critique which mainly states that fixed parameters in a model which does not adapt over time are bound to yield erroneous results. According to Lucas, macroeconomic models that treat certain parameters as fixed will produce misleading results. Lucas heavily criticized the models that were used to evaluate the effect of different policies, which assumed that parameter values would not change as a result of the policies being evaluated. In other words, treating parameters as stable in econometric theory will produce misleading and inaccurate policy advice. As a solution, Lucas critique advocated dynamic models for econometric analysis (Lucas 1976).

The essence of Markov random fields, or equivalently Gibbs random fields, assures that each entity is continuously affected by the state of the entire community, and therefore, the concerns with respect to invariance of the Lucas critique can be laid to rest. The Markovianity property simplifies the modeling of such an effect by stating that the influence of the entire community passes to an entity through its neighbors. In other words, the effect of the entire community is fully summarized in the state of the neighbors of an entity. The details are described in Sect. 2.

On the other hand, while the parameters of such influences among the entities is stationary in our models, it is quite logical to assume that such statistical parameters describing those influences are slow-varying in nature, and therefore, may be kept constant for a quite long run. However, if this is not satisfactory (as Lucas might have objected (Lucas 1976)), it is also possible to vary these parameters in an adaptive manner, but still slowly compared with the speed of change of the states of entities that continuously and quite rapidly affect the behavior of each entity. In that sense, our framework can be adapted quite easily to accommodate time-varying parameters, and thus satisfy the main tenets of the adaptive approach implied by the Lucas critique (Lucas 1976).

The presented GRF structure, and the developed simulator, can be effectively used to clearly observe the macro effects of simple few model parameters that describe the local entity interactions. It may be impossible, or very difficult at best, to establish those links between local interaction parameters and the subsequent macro results, analytically. Using the theoretical GRF model, and running the simulator, a policy maker can successfully test the macro results of a given set of local interaction parameters, and thus, can identify the local parameters that yield the desired macro results, and then, could direct its efforts to implement those local behavior patterns that match the desired parameters.

Even though we provide a concrete and complete structure to model economic activities, and state that just a few and simple parameters may be quite successful in mimicking real life situations, we do not attempt to fine tune those parameter values to fit actual empirical economic data. A reason for this is the obvious variability of those parameters for different types of collections of entities that make up the aggregate of interest: the nature of mutual interactions could be different in different societies; may change with respect to time based on the cultural changes, as well as, 
underlying technologies which provide the infrastructure for such mutual interactions; furthermore, those parameters will be quite different when modeling, for example, masses of consumers compared to financial networks consisting mainly of banks.

Furthermore, we do not attempt to compare our model with other models existing in the literature in terms of their success in modeling real data. Instead our focus is to provide a successful test-bed that links local level causes to their macro level results.

Even though we proceed with a simple model after providing the preliminary theoretical basis, it is not difficult to extend the work, including the associated simulator, to include heterogeneous entities into the network. Indeed, this would be an interesting case since it will provide a better model to the actual economic flora. A good feature of the presented model is its flexibility to easily handle such cases, as well. The price paid will be the associated complexity due to a larger set of parameters.

The contributions of this paper, beyond the state-of-art, can be summarized as, (i) a statement that Gibss random fields (or equivalently Markov random fields) can successfully model economic activities by linking simple micro-level entity interaction patterns, which can be represented by a few simple parameters, to their collective macro-level results, (ii) a related follow up statement which indicates that such simple GRF models can be used to predict consequences of micro-level financial network interactions to their macro-level consequences; this will provide a tool to early detect and thus maybe prevent financial network catastrophes, (iii) a full scale simulator that allows the users to input few GRF parameters that represent local interaction patterns in a network of entities, where each such simple entity can produce, consume and store wealth, and furthermore, engage in loan activities, and then test the eventual macro level status in such a network; the simulator can handle network sizes upto hundreds of thousands of entities, (iv) a tool in the form a theoretical GRF model and its implemented simulator that can be used to construct and test economic policies to induce desired macro-level end results.

The simulation examples included in this paper also provide answers to some common research problems, while showing the effectiveness of the provided theoretical Gibbs random field structure and the associated simulator. For example, we tested, via a set of simulations, the effects of network topology and strength of local interactions to the robustness of a network of economic entities which do produce and consume, and at the same time, engage in loan granting and receiving activities. Assuming statistically balanced aggregate consumption and production levels for the society, we formed two simultaneous neighborhood structures which represent both the social influence on consumption/production levels of entities and the connectivity of the allowed loan/debt network. And we observed, and came to a conclusion that a fully connected loan network is more robust in terms of debt defaults compared to a ringconnected sparse loan network, as expected, and in line with the results presented in Acemoglu et al. (2015). But we also observe and conclude that, this difference in robustness is quite small if the underlying economic behavior influences are restricted to a sparse ring-connected Gibbs model. On the other hand, if the underlying Gibbsian network has a fully-connected structure, where everybody directly influences everybody else's economic behavior, the parallel running fully-connected debt/loan network is significantly more robust compared to its ring-connected counterpart. We also observed an interesting, but rather expected by-product result: if the statistical 
interactions among the entities in the fully-connected Gibbsian economic network are too strong, while the entire society converges to total prosperity in most of the cases, occasional total extinction is also observed. In other words, strong statistical interactions result in a domino effect, where a total collapse resulting in an extinction is still possible, even though such a result has a low probability compared to total prosperity, in a fully connected debt/loan network. Further tests revealed that relative weakening of the interactions significantly reduced the probability of such extinctions; we did not observe any such extinction in our simulations, after such modifications. This observation provides a guideline for a wide-spread prosperity in a society: balanced aggregate consumption and production levels, together with well-connected but not too strong economic behavioral influences and well-extended loan/debt interactions among the entities lead to total prosperity of all entities.

A literature review is presented next. The mathematical preliminaries are presented in Sect. 2, a simple Gibbs random field (GRF) model for economic activities is introduced in Sect. 3 and an example is given in section 4. Applications to financial networks is discussed in Sect. 5. A complete simulator is developed; the simulator is described in Sect. 8, and some results obtained using this simulator are presented in Sect. 9. Finally, some conclusions are drawn in Sect. 10.

\subsection{Literature Review}

There is a large number of papers in the literature on financial network topologies and contagion of crises in financial networks, to which we cannot possibly do justice within a limited space. Nonetheless, we briefly touch upon those contributions in the economics literature that appear to be related to our goals in the present paper.

Leitner develops a model for financial networks to examine the spread of contagion based on the linkages, and conclude that while spreading contagion, linkages also induce bailouts, where liquid banks bailout illiquid banks to avoid contagion (Leitner 2005). Acemoglu and Ozdaglar propose a framework to understand the relationship between the financial network architectures and the probability of systemic failures due to contagion of risks (Acemoglu et al. 2015). They find out that the number of interbank connections is important, and show that there is a phase transition based on that number. They discuss the stability of such systems, and find out that while well-connectedness enhance the stability of the system under fewer and small negative shocks, beyond a certain point, such dense connections facilitate propagation of shocks and result in a more fragile financial system. Bech and Atalay study the topology of the federal funds network (Bech and Atalay 2010). They represent the federal funds market as a network where financial institutions constitute the nodes and the loans form the directed links; the link weights are the values of the loans between the counterparties. Using a transaction-level data set spanning 1997-2006, they analyze the characteristics of the overnight federal funds network and its evolution. Their empirical findings suggest that the network is sparse, which contributes to the magnification of contagion effects. Diebold and Yilmaz propose several connectedness measures for financial network topologies, and they use variance decompositions used in the network literature, for that purpose (Diebold and Yilmaz 2014). They show 
that variance decompositions define weighted, directed networks, so that the connectedness measures are related to similar measures used in the network literature. Gofman studies efficiency and stability of a financial architecture with too-interconnected-tofail institutions, and gives a network-based model of the over-the-counter interbank lending market in the US (Gofman 2017). He shows that trading efficiency decreases with the sparsity of interconnectedness due to lengthy intermediation chains.

Malamud and Rostek propose an equilibrium model of decentralized markets where general markets coexist with exchanges (Malamud and Rostek 2017). Afonso et al. (2011) focus on the effects of liquidity hoarding and the associated risks in the US overnight interbank market during the financial crisis of 2008. Their findings imply that counterparty risks are more important than the liquidity hoarding effects. Amini et al. (2016) in a study on resilience to contagion in financial networks obtain results for the magnitude of contagion in a large counterparty network and give an analytical expression that links network characteristics to asymptotic fraction of defaults. Afonso and Lagos (2015) focus on trade dynamics for federal funds and develop a model where banks have to look for a counterparty and then negotiate the size of the loan and the terms of repayment. They discuss the market dynamics for the reallocation funds and efficiency of such reallocations.

Zawadowski (2013) proposes a model of a financial system where banks hedge their portfolio risks using over-the-counter contracts. In their model, banks choose not to hedge counterparty risk, and therefore, the idiosyncratic failure of a bank can lead to systemic problems.

Glasserman and Young (2016) also study contagion in financial networks based on the level of interconnectedness of the financial system and its contribution to systemic fragility; they also give an excellent literature review related to this topic. Their work focuses on the interaction of the network structure with other relevant variables. They proposed various metrics to evaluate the susceptibility of the system to contagions.

Peltonen et al. (2014) study the network structure of the credit default swap (CDS) market and its determinants based on empirical data. Gofman (2012) explores the effects of intermediation on over-the-counter markets and their efficiency in the allocation of resources. Bargigli et al. (2015) study the multiplex structure of interbank networks.

\section{The Gibbs Random Field Model for Economic Activities}

\subsection{Mathematical Preliminaries}

We will use undertilde to describe random variables; no undertilde will be used for numerical values that these random variables take. We will use bold fonts to represent vectors (arrays). For example, $P(\mathbf{x}=\mathbf{x})$ means the probability that the vector random variable $\mathbf{x}$ takes the specific vector value $\mathbf{x}$. We will also use the notation $P_{\mathbf{x}}(\mathbf{x})$ for the same purpose, whenever we feel this notation is more appropriate. Indeed, whenever there is no ambiguity in the meaning, we will also use the shortened notation $P(\mathbf{x})$ to represent the same probability as described above. 
Markov random fields (MRF) are convenient and powerful in modeling intermingling of a large number of random variables. On the most complicated side, one can model a collection, $\underset{\sim}{\mathbf{X}}$, of random variables, ${\underset{\mathbf{x}}{i}}_{i}, i=1,2, \ldots$, by the joint probability density function (pdf) of all of these $\mathbf{x}_{i}^{\prime} s$. However, such a pdf cannot be directly constructed neither by observation, nor by deduction, especially when the number of variables is large; even simpler economic systems fall into this category. MRFs provide a very convenient and applicable tool to induce such complicated joint pdf's. The Markovian property assumes that the statistical interaction of a random variable, with the rest of the random variables, is perfectly summarized by its interaction with only those random variables in a defined neighborhood (for simplicity, we assume that the random variables take discrete values, and therefore, directly use the probabilities instead of pdf's):

$$
P\left(\mathbf{x}_{i}=\mathbf{x}_{i} \mid \mathbf{x}_{j}=\mathbf{x}_{j}, \forall j \text { such that } j \neq i\right)=P\left(\mathbf{x}_{i}=\mathbf{x}_{i} \mid \mathbf{x}_{j}=\mathbf{x}_{j}, j \in \eta_{i}\right), \quad \forall i .
$$

The index $i$ labels the random variables; usually it is interpreted also as the identifier of a specific spatial "location". The neighborhood, $\eta_{i}$, is nothing but a set of indices (locations) which excludes $i$. Usually, the neighborhood, $\eta_{i}$, for entity $i$ is rather small, and therefore, the model is not complicated. However, the model is still very powerful since it is proven that the conditional probabilities of each random variable, as described by Eq. 1 uniquely determine the joint probability of the entire collection $\mathbf{X}$ (Onural 2016). Unfortunately, that joint probability is almost impossible to compute; however, such explicit computations are usually not needed, anyway; ratios of such probabilities are usually adequate and such ratios are easy to compute.

Another mathematical result forms the link between Gibbs random fields (GRF) and the MRFs. It is proven that if $P(\mathbf{X}=\mathbf{X}) \neq 0$, for all possible realizations $\mathbf{X}$, then one can construct an equivalent MRF model for any GRF, and vice versa (Hammersley and Clifford 1971; Besag 1974; Spitzer 1971; Isham 1981). Since the condition, which only requires the prohibition of zero probability realizations (i.e., impossible realizations), is usually satisfied, the GRF-MRF equivalence can be used in many cases. Recently, it is also shown that this positivity condition is not necessary for the GRF-MRF equivalence, and constrained GRF's can still be employed with confidence while still enjoying the associated benefits (Onural 2016). A benefit that follows is the mathematical ease in understanding, defining, and dealing with GRFs, instead of more difficult MRFs.

With the above mathematical observations, we construct our model as follows: Each economic entity is labeled by an integer index $i$, and we associate a vector random variable $\mathbf{x}_{i}$ to each entity $i$. The elements of this vector represent separate economic variables associated with the entity $i$. For example, $\mathbf{x}_{i}=\left[p_{i} c_{i} s_{i}\right]^{T}$ may denote the production $p_{i}$, consumption $c_{i}$ and the accumulated wealth $s_{i}$ of entity $i$; the number of elements of the vector $\mathbf{x}_{i}$ and the definitions and other attributes of those elements depend on applications. To track the time variation of these variables, we also introduce another index $t$ in addition to the entity label $i$, so that we have $\mathbf{x}_{i, t}$. For simplicity, we will assume a discrete time index represented by integers, and therefore, we will have a discrete vector time series for each entity $i$. 
A Gibbs random field $\underset{\sim}{\mathbf{X}}$ has a joint probability as follows: We first decompose the set of all entities into subsets. We start with all subsets with one element, and then we keep on adding all subsets with two elements, three elements, and so on, until we reach the subset that includes all of the entities. Therefore, the elements of these subsets are collections of distinct entities and called "cliques" in the literature (Besag 1974; Isham 1981; Derin and Kelly 1989). Then, for each such subset, we assign a function which maps the realizations over that subset to a real number; we call those functions as "clique potentials", again, as usual in the literature (Isham 1981; Derin and Kelly 1989). There is no restriction on these potentials except the presented structure which requires that such potentials should be, and can only be, assigned to described cliques. Incidentally, this simple restriction assures that the corresponding equivalent MRF is consistent, and thus completely solves the otherwise complicated consistency issue of the conditional probabilities as described by Eq. 1 as they induce a global joint probability; this problem is quite difficult if attacked using the MRF formalism only, without establishing the MRF-GRF equivalence. Sum of all such clique potentials is called the total (Gibbs) energy, $U(\mathbf{X})$, of the realization $\mathbf{X}=\mathbf{X}$. And finally, the joint probability of $P(\mathbf{X}=\mathbf{X})$ is given by $k e^{-U(\mathbf{X})}$, where $k$ is just a normalization constant to assure that the sum of all probabilities over all realizations is equal to one. What makes the computation of these probabilities difficult is the computation of that constant $k$; however, algorithms that utilize the Gibbs model commonly utilize the ratios of the probabilities of different realizations, and therefore, the constant $k$ cancels out, and thus, disappears form the equations that describe the related algorithms.

Naturally, the total number of cliques for a given collection of $N$ entities is $2^{N}$ which is a prohibitively large number for any practical purpose. However, in most practical applications, a vast majority of clique potentials are set to zero; only a handful of clique potentials contribute to the total energy. This sparse nature of clique potentials also results in extremely efficient calculations of ratios of probabilities (Onural 2016).

Most of the Gibbs models in the literature are for scalar random variables over sites; here we have a vector random variable. This is not a problem, at all, in terms of the underlying theory. Indeed, if needed, one can further detail the nature of the interactions among the arbitrary scalar elements of the entire vector field, simply by converting the $N$-site vector field to an $k N$-site scalar random field for vectors with $k$ elements, each. An example of vector Gibbs random fields can be found in Onural (1988).

A simple, but still a powerful Gibbs model may assign potentials to each single entity (one element subsets) as $V_{i}\left(\mathbf{x}_{i}\right)$; if the collection is statistically homogeneous, $V_{i}$ becomes independent of $i$, and thus, can be denoted simply as $V$. We can then define two element clique potentials as $V_{\{i, j\}}\left(\mathbf{x}_{i}, \mathbf{x}_{j}\right)$; please note that, consistent with the set definition, the elements in a clique are always distinct, i.e., $i \neq j$, and therefore, to interchange $i$ and $j$ will result in the same clique. A commonly used practical model assigns nonzero $V_{\{i, j\}}$ only if $j$ is in the neighborhood of $i$; incidentally, the definition of the neighborhood assures that if $j$ is in the neighborhood of $i$, then $i$ is in the neighborhood of $j$ (Hammersley and Clifford 1971; Besag 1974; Spitzer 1971; Isham 1981). Again, for homogeneous (spatially stationary) random fields, $V_{\{i, j\}}$ depends only on relative positions of the locations $i$ and $j$. Economic entities may or may not be associated with physical spatial locations; therefore, other interpretations of 
homogeneity are needed and useful. Higher order cliques with three or more locations are rarely assigned nonzero potentials in applications. Surprisingly, even such simple Gibbs models which assign nonzero potentials only to single element and near-located double element cliques are quite adequate in modeling various natural and social stochastic interactions, successfully.

When the behavior of entities, or the nature of interactions among the entities, cannot be modeled by the same probabilistic models, in other words, when the overall system is not statistically homogenous, it is feasible, and actually not too difficult, to incorporate heterogeneous entities into the network. However, this will increase the complexity.

Incorporation of constraints, whether local or global in nature, is also possible, and does not violate the Markovian nature of the GRF, as shown in Onural (2016); such random fields are called constrained GRF's (CGRF's).

\section{A Simple GRF to Model Economic Networks}

We define an economic entity as a unit which is capable of producing, consuming and storing economic value. The entity is labeled by an integer index $i$, and therefore, the set of entities constitute a countable set. We will define three real-valued variables $p_{i}, c_{i}$, and $s_{i}$ to represent the level of production, consumption and storage associated with entity $i$. Naturally, all three variables are functions of time. Therefore, in most explicit form, the main economic activity of entity $i$ is represented by a vector function $\mathbf{x}_{i}(t) \triangleq\left[p_{i}(t) c_{i}(t) s_{i}(t)\right]^{T}$. For the sake of simplicity, and for subsequent discrete simulations, we will assume that the time variable is also discrete, and therefore, $t$ is an integer representing the time index of the discretization instants. Therefore, an equivalent alternative notation is $\mathbf{x}_{i, t} \triangleq\left[p_{i, t} c_{i, t} s_{i, t}\right]^{T}$.

The three components of the vector $\mathbf{x}_{i, t}$ which represents the time-varying economic activity of an entity are defined as follows: The production, $p_{i, t}$ is the sum of the worth of all economic activities of entity $i$ at instant $t$ that increase the wealth. Therefore, it includes plain production, as well as, any rent, interest, or any other form of income. Similarly, the consumption, $c_{i, t}$ is the sum of the worth of all activities of entity $i$ at instant $t$ that decrease the wealth, and that includes the debt service, if any, as well. Surely, any consumption can also be considered as a negative production, and vice versa, and therefore, one of the vector elements (either $p$ or $c$ ) can be eliminated; however, it is convenient to represent these two inverse activities with two separate variables since their statistical neighborhood dependencies are likely to be different, and therefore, they may have different roles in the GRF model that we construct. Indeed, if needed, the entity variables could be further partitioned to model more complicated business-type entity behavior, by defining other variables associated with further details. For example, to match the commonly used income statement tables used in standard accounting practices, the consumption variable may be partitioned to track the "cost of goods produced", $c_{i g}$, and the "overhead costs", $c_{i o}$, where $c_{i}=$ $c_{i g}+c_{i o}$. If this detail is incorporated into the model, then, naturally, $c_{i g}$ will be strongly correlated with $p_{i}$, whereas, $c_{i o}$ will have much less correlation; therefore, the statistical associations of these components will be different to reflect a more 
realistic model. However, this will complicate the model and the related simulations. We will not incorporate these possible details into the model, to keep it simple, at this time. Obviously, there will be constraints of local and global nature within the constructed GRF model to reflect the nature of the underlying economic facts. For example, $s_{i, t}$, which represents the accumulated wealth (net worth) of entity $i$ at time instant $t$, and the production and consumption levels are related as,

$$
s_{i, t+1}=s_{i, t}+p_{i, t}-c_{i, t}
$$

which is a very strong local constraint. Clearly, $s_{i, t}$ is a state variable, and the system is also one-step Markovian in time. Even though the model which tracks the consumption, production and storage activities of entities is complete, as also briefly mentioned before, for various reasons a more detailed version which further decomposes these variables, may be needed. For example, due to its special nature, a new debt variable, $d_{i}$ may be introduced by further decomposing the storage parameter as,

$$
s_{i}=a_{i}-d_{i}
$$

where $d_{i}$ represents the total debt owed by the entity $i$, and therefore, the newly generated variable $a_{i}$ is the rest of assets other than the total debt which is a negative asset. The variable $d_{i}$ may be positive, which represent net debtor; but, it may also be negative, and in that case, indicates that entity $i$ is a net creditor. The debt variable $d_{i, t}$ is also subject to non-local constraints since there are at least two parties in any debt/credit related activity: one who receives the loan and the one who grants the loan. In a simple isolated two-party loan arrangement, say between entities, $i$ and $j$, the constraint is, $d_{i, t}=-d_{j, t} \forall t$. In a society with more complicated debt/credit behavior, the constraint could be,

$$
\sum_{i \in B_{k}} d_{i, t}=0 \quad \forall t
$$

where $B_{k}, k=1,2 \ldots K$ form a partition for the set of all entities $B$; i.e., $\bigcup_{k} B_{k}=B$ and $B_{k} \cap B_{l}=\varnothing \forall k, l$, where $B_{k}$ represents a loan-wise closed group of entities where all debt/credit activities are conducted within the group; none of the members of $B_{k}$ can receive loans from or grant loans to outsiders. Clearly, the largest of such a set is the collection of all entities, $B$.

We will omit the time dependence of these variables in the notation whenever there is no ambiguity. The accumulated wealth as represented by the storage parameter, $s_{i}$, is also commonly referred to as the "net worth", or as the "owners equity" in different contexts. We have already indicated that $s_{i}=a_{i}-d_{i}$; indeed, any two of these three variables, $s_{i}, a_{i}$ and $d_{i}$, may be designated as the primary variables since the third variable is algebraically linearly dependent to the other two. If needed, the consumption variable $c_{i}$ can be further partitioned to explicitly indicate the debt service (interest paid) $b_{i}$, for example, in the form $c_{i}=g_{i}+b_{i}$, where in that case, $g_{i}$ represents the consumption other than the interest payments. Similarly, if there are any interest payments received as a consequence of a granted loan to another entity, this can also be explicitly indicated by replacing $p_{i}$ by $h_{i}+r_{i}$ where $r_{i}$ represents 


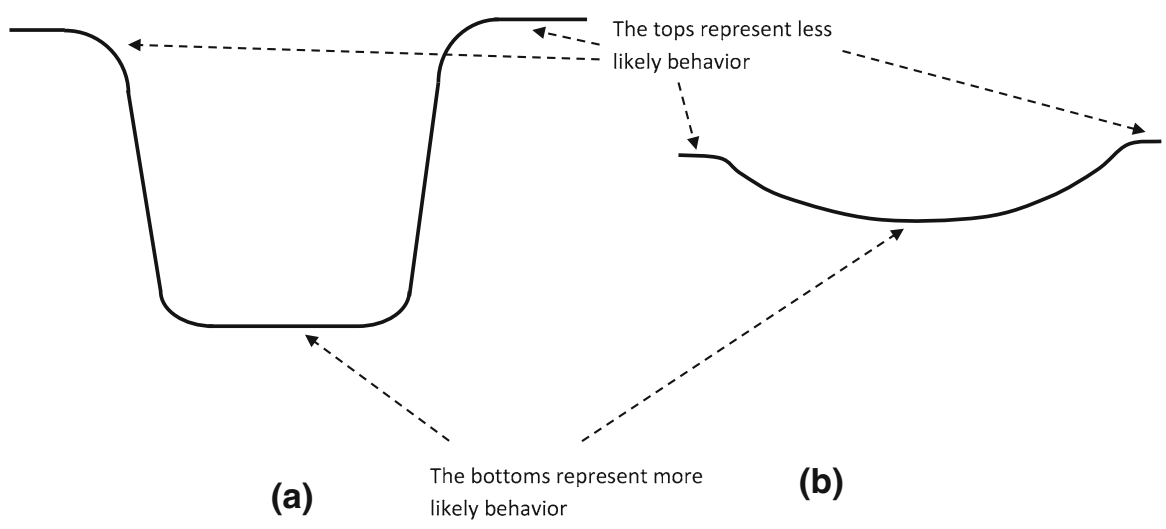

Fig. 1 A simple single clique potential may be designed to impose a rather unlikely behavior outside of the given limits by adopting steep walls, or $\mathbf{b}$ a more flexible behavior which allows more frequent deviations from the likely outcomes

received interest, and therefore, $h_{i}$ represents the rest of the production. We will keep the model simple, and therefore, choose not to incorporate these details.

We start building our GRF model by specifying the energy function as,

$$
U(\mathbf{X})=\sum_{i} V_{i}\left(\mathbf{x}_{i}\right)+\sum_{\{i, j\}} V_{\{i, j\}}\left(\mathbf{x}_{i}, \mathbf{x}_{j}\right)
$$

where $V_{i}\left(\mathbf{x}_{i}\right)$ and $V_{\{i, j\}}\left(\mathbf{x}_{i}, \mathbf{x}_{j}\right)$ represent, single and double site cliques, respectively. Therefore, we assume that all other higher order cliques have zero potentials. Since the site variable $\mathbf{x}$ is a vector, the model is still quite involved.

The specifications of $V_{i}\left(\mathbf{x}_{i}\right)$ and $V_{\{i, j\}}\left(\mathbf{x}_{i}, \mathbf{x}_{j}\right)$ must represent the natural behavior of an economic entity to get meaningful results. For example, if the entity is a single person, or if it is a simple household, $V_{i}\left(\mathbf{x}_{i}\right)$ should be specified to reflect unavoidable physical consumption needs of the entity for survival, and probably some additional intrinsically hard to avoid consumption needs of the entity. It should also reflect selfinitiated motivation for productivity and self-initiated tendencies for storage. It could also model intrinsic tendencies associated with borrowing habits. Considering these observations, one can conclude that this single clique potential will have a multidimensional well-shaped form where the bottom represents the more likely outcomes. The slope of the walls represent how flexible the deviations from those more likely outcomes; this slope should be steep for less flexible behavior. A representative figure for the one-dimensional case is depicted in Fig. 1.

One may first attempt to further simplify the single clique potential function by specifying that the components of the vector $\mathbf{x}_{i}$ to be statistically independent (separable $V_{i}\left(\mathbf{x}_{i}\right)$ in terms of its components). However, that might not be realistic. A more realistic specification will probably satisfy some qualitative requirements as follows: (i) Production, $p_{i, t}$ is strongly correlated to $c_{i, t}$, since a prudent entity will adjust its consumption and production levels to be similar. (ii) $p_{i, t}$ is expected to have a negative correlation with the storage parameter (accumulated wealth) $s_{i, t}$ since a wealthy 
entity does not need to produce to satisfy its consumption habits. (iii) It may also be expected that the level of production is also positively correlated to the amount of debt; indeed, in line with the famous saying "Debt is the horsewhip of the brave" (Borç yiğidin kamçısıdır.”); however, this may not be correct since maybe what generates the debt is habitual inadequate production levels; in this case, $s_{i, t}$ and $p_{i, t}$ will be negatively correlated, but obviously this is not a sustainable behavior. (iv) The consumption parameter, $c_{i, t}$, could be positively correlated with the storage parameter, $s_{i, t}$, since a wealthy entity is expected to have higher consumption levels. (v) With the same reasoning, the consumption, $c_{i, t}$, is expected to have a negative correlation with the debt variable, $d_{i, t}$, since it will be prudent for an entity to save and thus reduce the large debt; but there might be cases where this might not be correct, since ability to receive more debt may also boost the consumption for some entities.

Naturally, it is more difficult, but also more interesting to specify the pairwise clique potentials, $V_{\{i, j\}}\left(\mathbf{x}_{i}, \mathbf{x}_{j}\right)$. Based on common sense and observations, it may be quite logical to assume the following general statistical behavior patterns among the neighbors: $p_{i}$ has a strong positive correlation with neighbors' production levels; $c_{i}$ has a strong positive correlation with neighbors' consumption levels; $s_{i}$ has a deterministic algebraic dependence on production and consumption levels of the population, as indicated by Eq. 2 .

We restrict the direct interactions in our GFR model to only those outlined above, where only direct interactions are either among the components of $\mathbf{x}_{i}$ (we call these intra-site couplings), or among the corresponding components of neighbor sites (in other words, $p_{i}$ couples with $p_{j}$ 's only, $c_{i}$ couples with $c_{j}$ 's only, etc.) (we call these inter-site couplings). More complicated models are possible but marginal benefits of such complicated models may not justify the additional burden due to increased complexity.

Naturally, the introduced model parameters reflect the psychological foundations of economic behavior. A smaller $V_{\{i, j\}}\left(\mathbf{x}_{i}, \mathbf{x}_{j}\right)$ indicates a stronger desire and tendency between entities $i$ and $j$ to be similar to each other. Furthermore, $V_{i}\left(\mathbf{x}_{i}\right)$ reflects the self-satisfaction of entity $i$ as a consequence of its own economic state and behavior; a smaller $V_{i}\left(\mathbf{x}_{i}\right)$ represents a more satisfying, and therefore, a more likely state. A primary perception that affects the economic behavior of an entity is associated with the feeling of personal social/economic security; those who feel insecure about their future economic well-being tend to store (save) more.

\section{A Simple Example}

This simple example assumes a homogeneous network where the statistical behavior of all entities are the same, and therefore, the potential functions $V_{i}(\cdot)$ associated with each entity $i$ are identical for all $i$; subsequently, the index $i$ may be dropped from the potential functions for a simple notation. This assumption we adopt for this simple model may not correctly reflect real-life economies since a typical society includes quite differently behaving entities with totally different intentions and capacities. There is no need to specify a component for the potential function due to the storage variable $s_{i}$ since these values for all times are fully specified in a deterministic manner as 
indicated by Eq. 2 from the time sequences of $p_{i}$ and $c_{i}$. In this simple example, we specify $V\left(\mathbf{x}_{i}\right)$ as,

$$
V\left(\mathbf{x}_{i}\right)=V_{p}\left(p_{i}\right)+V_{c}\left(c_{i}\right) .
$$

More realistic models should combine these components in more complicated ways. We can then further specify the two components given by Eq. 6 . The consumption component $V_{c}\left(c_{i}\right)$ should represent and depend on self-initiated needs. This includes physical needs, as well as habitual consumption behavior. We propose a single clique consumption potential in the form of a binary function for simplicity, as follows:

$$
V_{c}\left(c_{i}\right)= \begin{cases}k_{c 1} & \text { if } c_{i} \in\left[T_{c 1}, T_{c 2}\right] \\ k_{c 2} & \text { else }\end{cases}
$$

where $k_{c 2}>k_{c 1}$. Such a choice will increase the probability of those values of $c_{1}$ that are in the specified interval $\left[T_{c 1}, T_{c 2}\right]$ relative to the probability of those values which are not in this interval. The probability of a value within the interval is uniform, as well as the probability of a value outside the interval, as a consequence of this binary form. As expected, $T_{c 1}$ and $T_{c 2}$ are external parameters and should be chosen to describe the likely range of self-initiated needs; similarly, $k_{c 1}$ and $k_{c 2}$ values, and especially the difference between them, will specify the relative likelihood of rather rare out of interval realizations compared to more likely realizations within the interval.

The production related component of the single clique (self-initiated) potential is also chosen to be similar to the consumption component which is defined above. Here the assumption is that the self-initiated production levels do not really show a large variation from one instant (period) to another (when for example the time interval is taken as a day or a longer period). Therefore, we specify,

$$
V_{p}\left(p_{i}\right)= \begin{cases}k_{p 1} & \text { if } p_{i} \in\left[T_{p 1}, T_{p 2}\right] \\ k_{p 2} & \text { else. }\end{cases}
$$

Another specification is related to possible ranges of these three main variables. Obviously, both $p_{i}$ and $c_{i}$ should be non-negative. An alternative simpler model may combine these two variables into a single variable which might be properly named as the "net production" which is defined as $p_{i}-c_{i}$; in that case, all real valued outcomes could be possible.

In this simple example, we can explicitly define the neighborhood $\eta_{i}$ of entity $i$ as consisting of its a few "nearby" neighbors. The term "nearby" may or may not define a physical measure of closeness; however, it defines the labels of those entities which are interacting directly with the entity $i$. Please note that we are referring to "direct" interactions; indeed, all entities may still, and usually do, interact with each other indirectly via the consecutive intermingling of all entities via their intersecting neighborhoods. In other words, the Markovian property as implied by the Gibbs model through the MRF-GRF equivalence provides an interaction of an entity $i$ with all other entities, via its neighborhood. For a very simple interaction model, we define the neighborhood, $\eta_{i}$ of $i$ as the set $\{i-1, i+1\}$ for all $i$ 's. If the number of entities are finite and equal 
to $N$, we may define the set of all $i$ as integers in $[0, N-1]$, and in that case, the addition $i+1$ and the subtraction $i-1$ should be implemented as modulo $N$ arithmetic operations. Such a choice indicates a ring form of a network where each entity directly interacts only with its left and right immediate neighbors. We further assume that the economic entities adjust their economic activities to be similar to their neighbors. This assumption seems quite valid within the society; people, or households, tend to have similar consumption behaviors as their neighbors. This similarity may then induce similar levels of production activity, which in turn may induce similar storage results. This similarity may be quite strong or quite weak. However, it is not expected to have a negative correlation among the neighbor behaviors. With these observations and simplifying assumptions, we may choose to define the pairwise clique potentials as,

$$
V_{\{i, j\}}\left(\mathbf{x}_{i}, \mathbf{x}_{j}\right)=\mathcal{V}_{\{i, j\}, c}\left(c_{i}, c_{j}\right)+\mathcal{V}_{\{i, j\}, p}\left(p_{i}, p_{j}\right) \quad i \neq j,
$$

which implies no direct interaction between $p_{i}$ and $c_{i}$ components. More realistic models, however, may include those cross-coupling terms, as well. Again, a homogeneity (spatial stationarity) assumption allows us to drop the $i$ and $j$ subscripts and write the potential as, $V_{q}\left(\mathbf{x}_{i}, \mathbf{x}_{j}\right)$, where the new index $q$ represents only the clique shape (connectivity) and not its absolute location. Since our simple ring model has only one clique shape $\{i, i+1\}$ which represents direct interactions of only two sideby-side entities, we can further drop the index $q$, as well. We may then specify the components indicated by Eq.9, for our simple model, as,

$$
\mathcal{V}_{p}\left(p_{i}, p_{j}\right)= \begin{cases}v_{p 1} & \text { if }\left|p_{i}-p_{j}\right| \leq T_{v p} \text { and if }|i-j|=1 \\ v_{p 2} & \text { if }\left|p_{i}-p_{j}\right|>T_{v p} \text { and if }|i-j|=1 \\ 0 & \text { else, }\end{cases}
$$

where $v_{p 1}<v_{p 2}$. We used $\mathcal{V}$, which labels a two-variable function $\mathcal{V}(\cdot, \cdot)$, to distinguish it from the single clique potential function $V(\cdot)$, which is a single-variable function, when the label indices are omitted in the homogeneous field cases. The implications of this chosen form is to assign higher probabilities to those patterns $X$ in which the production level of an entity is similar to that of its two neighbors. Therefore, those $X$ 's with frequently unmatched production levels among the two consecutive entities within the formed ring structure will have lower probabilities. The term "similarity" means that the difference is within an externally provided threshold level $T_{v p}$. The range defined by this threshold, as well as the difference between the values $v_{p, 1}$ and $v_{p, 2}$, will impose more relaxed or tight conditions to enforce the implied similarity conditions. The pairwise clique potentials associated with the consumption can also be defined similarly, as,

$$
\mathcal{V}_{c}\left(c_{i}, c_{j}\right)= \begin{cases}v_{c 1} & \text { if }\left|c_{i}-c_{j}\right| \leq T_{v c} \text { and if }|i-j|=1 \\ v_{c 2} & \text { if }\left|c_{i}-c_{j}\right|>T_{v c} \text { and if }|i-j|=1 \\ 0 & \text { else. }\end{cases}
$$


We will later show by simulations that even such a simple model could simulate some realistic cases, quite well.

The structure of direct couplings can be easily altered by defining larger neighborhood sets. In the extreme case, if the neighborhood of an entity consists of all other entities in the universe, then we achieve a model for a fully connected network.

This simple model in this example does not consider any debt/credit activity.

If, in case, $\mathcal{V}_{p}\left(p_{i}, p_{j}\right)=\mathcal{V}_{c}\left(c_{i}, c_{j}\right)$ then the model can be even further simplified by combining $p_{i}$ and $c_{i}$ as a single variable, for example, as $z_{i}=p_{i}-c_{i}$, and then proceeding as a scalar random field of variable $z_{i}$ 's; in that case, $z_{i}$ represents net production.

\section{Financial Networks}

A concern among the creditors is the timely collection of the loans they granted to borrowers on the maturity dates. As the loan activity is quite extensive and inter-related in a typical society, defaults may trigger large scale problems since they may quickly propagate. This creates a public concern and is usually called a crisis; public bodies often interfere and generate remedies in case such widespread defaults emerge. Furthermore, proactive regulations may be imposed to avoid such widespread defaults. For example, the systemic risk and stability of financial networks is studied in Acemoglu et al. (2015).

It is possible to extend the basic GRF model developed in Sects. 2 and 3 to understand the degree of vulnerability/robustness of a network based on violations of debt payment obligations. For this purpose, we include into the model a bookkeeping capability to explicitly keep track of debt obligations and the possible default of debtors, together with a default procedure. We define a loan as $\Delta_{i j t_{i} t_{m}}$ for the loan given by entity $j$ (creditor) to entity $i$ (debtor) at instant $t_{i}$ for the maturity instant $t_{m}$; the amount of the loan is $\Delta_{i j t_{i} t_{m}}$. Each such individual loan is recorded and such records are kept until maturity.

\subsection{Example: Homogenous Network}

In this simple case, we assume a homogenous network where all entities have the same statistical behavior; therefore, in terms of debt/credit relations, any entity is capable of granting (creditor) or receiving (debtor) loans. The simple rules we adopt are:

- Any entity $j$ may grant a loan to any other entity $i$, with no discriminating preferences.

- The interest is included in $p_{j}$ (received interest) or in $c_{i}$ (paid interest) (therefore, we choose not to keep explicit track of the interest components in $p$ or in $c$ ).

- Any asset, or part of it, in $a_{j}$ can be given as a loan. The total of loans to be granted at an instant of time by $j$ may not exceed the total assets $a_{j}$, at that time instant. Therefore, $-d_{j} \leq a_{j}$.

- Each loan activity obeys the conservation rule: $\Delta_{i j t_{i} t_{m}}=-\Delta_{j i t_{i} t_{m}}$. For each entity, $d_{i, t+1}=d_{i, t}+\sum_{j, t_{m}} \Delta_{i j t t_{m}}$. 
- We assume that there is no default on debt service in case of a positive $a_{i}$, i.e., interest payments are cost items and always paid if there are resources to do so, to simplify the example. Therefore, if $a_{i, t_{m}} \geq \sum_{j, t} \Delta_{i j t t_{m}}$ on the maturity instant $t_{m}$, all obligation $\Delta_{i j t t_{m}}$ are paid back by debtor $i$ to its creditors.

- If, however, $a_{i, t_{m}}<\sum_{j, t} \Delta_{i j t t_{m}}$ on the maturity instant $t_{m}$, then entity $i$ defaults. The default procedure is as follows: entity $i$ pays to its creditor all of its storage $s_{i, t_{m}}$ and disappears. Its neighbors, reorganize their neighborhood, based on a specified rule, when entity $i$ no longer exists. In case more than one creditor expects capital payments on the same maturity instant from a defaulting debtor, the storage $s_{i, t_{m}}$ of that debtor is distributed to those creditors in a prorated manner. Those receivables by defaulting entity $i$, due to loans granted by $i$ to others in the past, and therefore, included in assets $a_{i}$, are kept in the books until their maturity dates, and on the maturity date, they are paid, again in a prorated manner, to the past creditors of already defaulted $i$.

The default rule may be modified for more complicated cases. For example, instead of not allowing a negative asset entity to survive as in the example above, more relaxed rules, such as, $\sum_{i \in B_{k}} a_{i} \geq \sum_{i \in B_{k}} \sum_{j, t} \Delta_{i j t t_{m}}$ may be imposed to a group $B_{k}$ for survival. In this case, the group $B_{k}$ may still be accepted as healthy, since the total debt they owe is still less than their total assets. If this relaxed rule cannot be satisfied, all $i \in B_{k}$ default.

The simple model as introduced in Sects. 3 and 4 can be easily modified to match the features of even more complicated networks. For example, non-homogenous networks may be modeled by adding banks who may overwhelmingly dominate the loan granting activity to ordinary household entities. Companies whose loan behavior, and therefore, the model parameters are quite different from a household could be integrated into the model; typical distributions of different entities, or groups of entities may be incorporated. The proposed model and its associated structure are still quite simple and tractable even for such complicated modifications.

This simple model can also be used to investigate robustness of some financial network topologies.

We have based our system on a GRF model where there are statistical interactions among production and consumption activities of neighboring entities, as described in previous sections; that necessitated a neighborhood definition, together with related parameters. Here in this section, we have also included a debt/loan activity among the entities; such an extension necessitates one more neighborhood structure for such debt/loan activities. We define this second neighborhood as the "loan neighborhood", and impose a related constraint which restricts the entity $i$ to receive loans from, or grant loans to those entities in the loan neighborhood of $i$. The loan neighborhood also posses the simple properties of a MRF neighborhood: simply, $i$ is not in its own neighborhood, and if $j$ is in the neighborhood of $i$, then $i$ is in the neighborhood of $j$. In order to avoid any ambiguity, we label the intrinsic neighborhood structure of the GRF model as the "GRF neighborhood" whenever necessary; however, we will refer to that neighborhood simply as the "neighborhood" whenever there is no such ambiguity. 


\section{Hierarchic GRF Representation: Coarser Levels}

Consolidation of all of these three main variables, $p_{i}, c_{i}$ and $s_{i}$ among many entities is possible. Such consolidations define a hierarchical random field structure. Let us name the hierarchical levels as "finer" and "coarser" levels. The finest level is the level where each entity is in its simplest form, and cannot be further decomposed into smaller entities. The coarsest level is naturally the single entire collection of all entities, and therefore, represents the global total of production, consumption and storage. (The global debt sums to zero, by definition.) Let the integer index $l$ represent the level of coarseness, where $l=0$ is the finest level. Therefore, the consequence of consolidation at the finest level is

$$
\begin{aligned}
& p_{m}^{1}=\sum_{i \in S_{m}} p_{i}^{0} \\
& c_{m}^{1}=\sum_{i \in S_{m}} c_{i}^{0} \\
& s_{m}^{1}=\sum_{i \in S_{m}} s_{i}^{0} \\
& d_{m}^{1}=\sum_{i \in S_{m}} d_{i}^{0} .
\end{aligned}
$$

Here, the superscripts indicate the value of the level index $l$. The index $m$ represents the super-entity which emerges as a consequence of consolidation of entities underneath it; these finer level entities which are consolidated under a super-entity $m$ are defined by the set $S_{m}$; the elements of this set are simply the labels ( $i$ 's) at the $l=0$ level that collectively constitute the super-entity $m$ at level $l=1$. We will not use the term "super-entity" all the time; instead, we will use the simple term "entity" also for higher levels (for $l=1, \cdots$ ), whenever there is no ambiguity for the level being addressed. A requirement for the sets $S_{m}$ is that they are mutually exclusive for different values of the index $m$. Furthermore, the union of all such sets covers all elementary entities for all $i$. Therefore, $S_{m}$ 's constitute a partition.

A strong and useful observation is the fact that entire stochastic model at any higher level of coarseness (i.e., for $l=1, \cdots$ ), with all its structure and parameters, is fully specified by the stochastic model defined at the finest level, $l=0$. Therefore, although it is mostly the psychological factors that determine the behavior at the finest level (single person economic entities), it is the induced statistical patterns of those underlying psychological factors that determine the collective economic behavior patterns at coarser levels.

\section{Further Remarks}

It is worth noting that some closely observed economic and financial parameters and indicators that are commonly used by policy makers at macro and micro levels are essentially implicitly within the model provided in this paper, and therefore, can 
be deduced from a given GRF model. Those include prices, inflation rate, growth rate, interest rates, as well as other economic concepts like elasticity, etc. Actually, the strength of the proposed model is its ability to yield the statistical properties of both transient and steady-state global production and consumption levels (and other parameters), precisely, as indicated in Sect. 6, once the fine level model (structure and parameters) is known. Those statistics do include how global production and consumption levels are linked to each other; that surely includes deviations and similarities among global consumption and production behaviors. The statistics that describe such deviations and similarities are one-to-one related to probabilities, time-variations, and related features of inflation/deflation fluctuations. (Note: if global production, $p_{t}$, is greater than global consumption, $c_{t}$, then this is a situation of affluence. But if $c_{t}$, is greater than $p_{t}$, then there is scarcity.) The same observation is also true for associated financial results: those elementary parameters at the fine level do impose mechanisms and probabilities of global operations, including smooth cases as well as those disruptive cases that are usually referred as financial crises characterized by widespread defaults, etc.

\subsection{Some Proposed Economic Measures}

\subsubsection{Robustness}

A strong parameter to watch to understand the economic behavior of an entity and to make predictions about its future states is the rate of relative change of the storage, $s_{i, t}$. We define the rate of relative change as:

$$
r_{i}(t)=\frac{\frac{s_{i}(t)-s_{i}(t-\delta t)}{s_{i}(t-\delta t)}}{\delta t}
$$

Although it is tempting to let $\delta t \rightarrow 0$, and thus write a derivative to the righthand side, this can be done only over large aggregates at coarser levels where all time varying parameters may converge to continuous variables, or when it is known that these parameters are continuous for an entity. Otherwise, these discrete realizations of a stochastic process do not have derivatives. Naturally, those entities with negative $r_{i}(t)$ should worry, and must correct this unsustainable situation. Normally, nonnegative $r_{i}(t)$ indicates a "healthy" or "robust" economy, and the degree of "robustness" gets better as $r_{i}(t)$ gets larger. We will call parameter $r_{i}(t)$ as the robustness of entity $i$. Even though a significantly negative $r_{i}(t)$ is definitely a strong reason for "unhappiness" of an entity $i$ since it is quickly getting poorer, a similar statement cannot be made for nonnegative $r_{i}(t)$, since the "happiness" in that case may be a function of both $r_{i}(t)$ and $c_{i}(t)$; some entities can only be happy when their consumption is large and their wealth is not diminishing, on the other hand some entities may be perfectly happy with a small level of consumption as long as their wealth is steady or growing.

A closely related parameter which may be taken as an alternate measure of robustness is the ratio $s_{i} / \bar{c}_{i}=y_{i}$, where $\overline{c_{i}}$ denotes average consumption. A logical choice of average consumption is a weighted average of consumption $\overline{c_{i, t}}=$ 
$\frac{1}{N} \sum_{\tau=t-N+1}^{t} w_{\tau} c_{i, \tau}$ over a reasonable number of time instants $N$; weights, $w_{\tau}$, could give more emphasis to recent instants. Incidentally, such weighted averages are frequently used in the literature that examines habit formation in consumption (see, for example, Fuhrer 2000; Havranek et al. 2017). Fuhrer explores a monetary-policy model with habit formation for consumers, in which consumers' utility depends in part on current consumption relative to past consumption (Fuhrer 2000). The empirical tests developed in that work show that one can reject the hypothesis of no habit formation with tremendous confidence (Fuhrer 2000). Havranek et al. (2017) examine estimates of habit formation reported in published studies. A large $y_{i}$ represents a more robust entity with a large storage to accommodate current consumption habits, even if there is a problem with production abilities.

\subsubsection{Replenishment Difficulty}

This parameter reflects the aggregate of efforts to increase the storage (wealth) for an entity. We label it as $f_{i}$ and state it as a function of $r_{i}$. The actual form of the function depends also on the psychological factors at the finer levels, and on their induced statistical results at coarser levels. The time variation of the price parameter $f$ at the coarsest level implies inflationary $\left(\frac{\delta f(t)}{\delta t}>0\right)$, or deflationary $\left(\frac{\delta f(t)}{\delta t}<0\right)$ consequences of certain economic behavior patterns as outlined by the model parameters. We may also call this parameter real price.

\subsubsection{Economic Satisfaction}

It seems to be logical to define the overall economic satisfaction, $e_{i, t}$, of an entity $i$ at present time $t$ as the sum of total consumption in the past and the current storage, as $e_{i, t}=s_{i, t}+\sum_{\tau=-\infty}^{t} c_{i, \tau}$.

\subsubsection{Time-Varying Model Parameters}

The current model assumes time-invariant model parameters. Such a model is already quite powerful and already includes feedbacks due to massively interactive network where each entity affects every other connected entity. However, if this is not satisfactory, it is possible to vary these parameters in an adaptive manner; however, these GRF model parameters will change slowly compared with the speed of change of the states of entities that continuously and quite rapidly affect the behavior of each entity. In that sense, our framework can be adapted quite easily to accommodate time-varying parameters, advocated by Lucas in his critique (Lucas 1976). However, the model could be further improved by incorporating time-varying parameters in a meaningful manner; thus it could be possible to model an adaptive system. For example, prevailing macro results of a given system, like the price parameters $f_{i}$, as defined above, could influence the entity parameters, in turn. 


\section{The Simulator}

A computer program is developed to simulate the simple model given in Sect. 4; the entities can grant or receive loans, and therefore, the structure as described by Example 5.1 is included. The details of the simulator is given in its User's Manual (Firtına 2015). The simulator is developed in $\mathrm{C}++$ programming language with OpenGL and OpenCL libraries. A user friendly interface is also provided. The user defines the total number of entities at the beginning. Furthermore, a connectivity matrix, which defines the neighbors of an entity, is read from a stored file; the user should generate and store this matrix before the simulation starts. This matrix represents a graph which defines the neighborhood as imposed by the employed GRF model; we will call this matrix GRF Connectivity Matrix. Similarly, a loan neighborhood structure is imposed by defining the debt/loan neighbors of each entity by a loan connectivity matrix which should also be generated and stored by the user. If a loan connectivity matrix is not provided, then an entity can request a debt from any other entity in the entire network, as the default case. Both of these graphs are undirected, i.e., if entity $i$ is connected to entity $j$, then entity $j$ is also connected to entity $i$. All of the needed model parameters, $k_{c 1}, k_{c 2}, T_{c 1}$, $T_{c 2}, k_{p 1}, k_{p 2}, T_{p 1}, T_{p 2}, v_{c 1}, v_{c 2}, T_{v c}, v_{p 1}, v_{p 2}, T_{v p}$, as described in Sec.4, are entered by the user via the interface. The maximum maturity duration that an entity might request is also requested by the simulator interface.

The simulator uses random integer values to seed the underlying Metropolis algorithm. An option is provided to allow the user to locate a file in which random integer values are stored.

The simulator implements the Metropolis algorithm that evolves from a given state of the Markov chain to a steady-state where the probability of an emerging state (pattern) is as implied by the parameters of the Gibbs random field model (Beichl and Sullivan 2000). The simulator, therefore, is a Markov chain that evolves in discrete time instants; at each step, randomly selected values of variables $c_{i}, p_{i}, s_{i}$ and therefore resultant for a set of entities are collectively tested for a possible alteration, and the state is either kept as is, or altered as proposed. The selection of a test state for a possible transition requires care to satisfy the theoretical concerns that assure convergence to the prescribed steady-state distribution (Onural 1991, 2016). A range of possible discrete values are defined for each variable, depending on the nature of that variable. For example, $p_{i}$ and $c_{i}$ variables are restricted to integers in $[0,255]$; surely, these ranges may be modified to fit a certain simulation case, if needed.

The Metropolis algorithm evolves from state to state; each state is defined as the collection over all entities, of the specific values that the entire set of parameters of each entity is set to Beichl and Sullivan (2000). At each state, $\mathbf{X}=\mathbf{X}$, a transition to a new state, $\mathbf{X}=\mathbf{Y}$ is proposed. The probabilities of the proposed state and the current state are compared, under the imposed GRF model as indicated by the model parameters. Even though the probabilities are difficult to compute, the computation of the ratio, of those probabilities, $P(\underset{\sim}{\mathbf{X}}=\mathbf{Y}) / P(\underset{\sim}{\mathbf{X}}=\mathbf{X})$, is quite simple and straightforward. Let us denote this ratio as $r$; if $r>1$, i.e., if the proposed state $\mathbf{Y}$ is more likely than the current state $\mathbf{X}$, then the proposed transition is accepted (the chain changes its state). Otherwise, if $r<1$, the alteration is accepted with probability $r$; in other words, less likely states may also be accepted, but the probability of accepting such a transition is 
low in line with a low $r$. After a sufficient number of transitions, the outcome becomes a sampler from the implied Gibbs distribution. Metropolis algorithm and its many variants are well investigated in the literature (Beichl and Sullivan 2000).

For our simulator, the discrete evolution steps of the Metropolis algorithm do not necessarily represent equal real-time steps. Rather, it represents the real-time instant that the partition of entities does its consideration for an alteration of its variables (test instant). For the sake of fast convergence to steady state, as indicated by the model and its parameters, all of the entities are split into sets $P_{k}$ such that the collection of those $P_{k}$ 's form a partition: $\bigcup_{k} P_{k}=$ set of all entities, and $P_{k} \bigcap P_{l}=\varnothing$ if $k \neq l$; a set $P_{k}$ may include only those entities which are not in the neighborhood of any other entity in the same $P_{k}$. The simulator forms such $P_{k}$ 's at the beginning, and this is transparent to the user. The Markov chain evolves sequentially starting from $k=1$, progressing by increasing $k$ by one, modulo $K$, where $K$ is the number of sets in the partition. At each time instant, all of the entities in $P_{k}$ are subject to change randomly to form $\mathbf{Y}$, whereas, all other entities in all other sets $P_{l}, l \neq k$ are kept unchanged. Depending on the model parameters, transition to a tested state may be rather easy; in other words, accepted transitions do occur quite often, even in the steady state. On the other hand, model parameters may dictate quite stiff systems which rarely perform a transition, and therefore, quite persistent to keep its state.

The simulator also implements the hierarchical structure presented in Sect. 6. The user can interactively enter the indices of finer level sites which consolidate to form a super-entity at a coarser level. It is possible to observe the activity at any level of coarseness of choice.

The simulator output is a graphic output, which designates a location for an entity on the screen. Depending on the choice of a user, locations of the entities might be set either randomly or in a sequential order. The entity is represented as a simple geometric cubic shape located at that designated site. The brightness of that geometric shape represents the numerical value of the variable being observed, i.e. the storage value; high values are represented as greenish, whereas, low values are closer to red color, with a linear mapping of brightness for the range of the variable. User interface of the simulator allows a user to define minimum value for an entity that will be completely red if it has higher than or equal to that minimum value. Otherwise, an entity is assigned to a color between green and red. This minimum value can be changed dynamically as the simulator runs. Furthermore, if an entity goes into a default procedure, the cubic shape representing that entity disappears from the screen. A complete transcript of each and every test and its consequence may also be kept as a file, provided that the total number of entities, and the total number of transitions are reasonable; for a large network the computer disk capacity might not be enough to keep such a transcript especially for longer runs.

The simulator mostly makes use of parallel computation power of graphic processing units (GPUs) to calculate the formulas that are described in Sects. 3 and 4 for each entity. Thus, each random variable for an entity, decision for a default procedure, and calculations of $V_{i}\left(\mathbf{x}_{i}\right)$ and $V_{\{i, j\}}\left(\mathbf{x}_{i}, \mathbf{x}_{j}\right)$ for each entity $i$ and $j \in \eta_{i}$ are performed in parallel. This parallelism allows the simulator to be able to simulate a model that includes hundreds of thousands of entities while displaying the results in real time, which would not be possible with a standard CPU. 
For the simulation of economic networks with financial (debt/credit) activities, the Metropolis algorithm is modified to take care of the imposed restrictions (Onural 1991, 2016):

(1) At an instant $t$, group entities into $P_{k}$ 's such that each entity is included in exactly one such set $P_{k}$ and no two neighbor entities are in the same set $P_{k}$, i.e. if $i \in P_{k}$, and if $i \in \eta_{j}$, then $j \notin P_{k} \forall i \in S$, where $P_{k}$ is $k$ 'th set and $S$ is set of all entities; $k=1, \cdots, K$, where $K$ is the number of sets in the partition. The storage values of each entity is initialized by setting them to a default value, which is chosen to be high enough to assure that premature defaults are unlikely during the initial transition period; therefore, it can be assumed that the system reaches statistical steady-state before the defaults are observed.

(2) Set $k=1$.

(3) $\bullet \forall i \in P_{k}$ choose test variables $t e s t_{-} c_{i}$, test_p $p_{i}$ randomly, from uniform distributions.

- Compute the ratio, $r$, of the probabilities of the current state and the proposed next state above, using the proposed changes in the parameters as prescribed above (test_c $c_{i}$, test_p $p_{i}$ ). If $r>1$ accept the transition (the chain always evolves if the next state is more likely); if $r<1$ accept the transition with probability $r$ (the chain evolves with probability $r$ if the proposed state is less likely than the current state.)

- If the proposed next state transition is accepted, set the proposed test_c $c_{i}$, test_ $p_{i}$ values as the $c_{i}$ and $p_{i}$ values of entity $i$, respectively, $\forall i \in P_{k}$. Otherwise, $c_{i}$ and $p_{i}$ remain unchanged.

(4) If $k \neq K, k \leftarrow(k+1)$ and go to Step 3 .

(5) Update storage values, $s_{i}, \forall i \in S: s_{i}=p_{i}-c_{i}$.

(6) Check for all debt payments whose maturity date is this time instant $t$. If the assets of an entity allow such due debt payments, perform the payments. If however, assets of an entity are not sufficient to pay all such due debt, the entity defaults, and the default procedures are applied:

- If entity $i$ defaults, then;

- Pay all creditors using all of the net assets $s_{i}$ in a prorated manner. The future receivables of a defaulting entity $i$, as a consequence of loans granted to others by $i$ in the past, are kept in the books, and when the due date comes, creditors of the defaulting entity $i$ receive the payments, again in a prorated manner, provided that the debtor who will make the payments is still alive at the due date; if the debtor has also disappeared by defaulting in the mean time, such debts are totally erased from the books.

- Remove $i$ from the network.

- Form the new neighborhoods according to preset neighborhood rules.

- Rearrange the loan connectivity graph according to preset rules.

(7)

- Randomly form pairs $\{i, j\}$ where $j$ is in the loan neighborhood of $i$ (therefore, $i$ is also in the loan neighborhood of $j$ since we have a GRF model in place). 
Thus, all entities in a partition are paired for a possible loan activity; in case the total number of entities in the partition is odd, the last single entity does not participate in a loan activity. Choose randomly a $\Delta_{i j t t_{m}} \in[$ range $]$ from a uniform distribution. This is the possible new loan from $j$ to $i$ if $a_{j} \geq \Delta_{i j t t_{m}}$; if however, $a_{j}<\Delta_{i j t t_{m}}$, there is no any debt activity among $i$ and $j$. The maturity date, $t_{m}$, for any such loan is also randomly determined from a uniform distribution in the range 1 to $T_{m}$ where the maximum duration $T_{m}$ is an input controlled by the user at the beginning of each simulation and kept unchanged throughout the simulation.

- Update the loan parameters of $i$ and $j$ as $d_{i, t+1}=d_{i, t}+\Delta_{i j t t_{m}}$, and $d_{j, t+1}=$ $d_{j, t}-\Delta_{i j t t_{m}}$.

(8) Increment $t$ by one and go back to Step 1 until the simulator is stopped by an external user interrupt.

This algorithm assures that the constraint imposed by Eq. 4 is satisfied while assuring that a state reached after a sufficient number state transitions is a sample from the constrained Gibbs distribution implied by the chosen model parameters (Onural 2016).

Consideration of consumption, production and storage parameters, together with the debt parameter is essential to have meaningful results, since the ability to meet debt obligations is inherently related to the actual production and savings abilities and behaviors of entities. Each entity is provided with a reasonable amount of net worth (storage) $s_{i}$ at the beginning of the simulations; the starting $s_{i}$ is the same for all $i$ 's and good enough to assure that premature defaults, before the Metropolis iterations reach its steady state, are unlikely.

A feature of the simulator is an external button to pause the Markov chain at any instant. This allows modification of storage values of any entity externally; this feature allows to implement external shocks by explicitly reducing the storage of any entity. The chain is then started to evolve from its externally modified state to observe the associated transient and steady-state behavior. It is possible to implement many such modifications, one after another, by pausing the chain as many times as desired.

Depending on the hardware resources of the computer that the simulator is implemented, the number of entities in the network may go upto a few hundred thousands; this will allow to test realistic populations, if desired.

\section{Some Simulation Results}

Here in this section we run a few simple simulations; even though the simulations are simple, we can still draw some conclusions. The intention here is to demonstrate that the proposed model, and the associated simulator, can be used to draw such conclusions, provided that careful simulation based experiments are designed, and a statistically meaningful number of simulations are conducted. The entities and the neighborhood structures, and therefore, the associated network topologies in the three sets of simulations presented below are similar to those financial networks analyzed by researchers (see for example, Acemoglu et al. 2015). 
Table 1 Simulation 1 parameters

\begin{tabular}{cccccccccccccc}
\hline$k_{c 1}$ & $k_{c 2}$ & $T_{c 1}$ & $T_{c 2}$ & $k_{p 1}$ & $k_{p 2}$ & $T_{p 1}$ & $T_{p 2}$ & $v_{c 1}$ & $v_{c 2}$ & $T_{v c}$ & $v_{p 1}$ & $v_{p 2}$ & $T_{v p}$ \\
\hline-0.3 & 0.3 & 100 & 120 & -0.1 & 0.1 & 100 & 120 & -0.3 & 0.3 & 30 & -0.3 & 0.3 & 30 \\
\hline
\end{tabular}

Simulation 1 We set the number of entities to 20, and the Maximum Maturity Time as 10 . We set the GRF Connectivity Matrix to indicate simple ring-type connectivity; i.e., each entity is statistically directly influenced only by its left and right (total of two) neighbors. We also defined the Debt Connectivity Matrix to have the loan activity restricted to those immediate two neighbors only. Therefore, both the statistical interactions and the loan/debt activity has a ring connectivity. The parameters are set as in Table 1.

Therefore, these parameters describe a network of 20 entities where each entity is,

- quite likely to have intrinsic (self-generated) consumption between 100 and 120 units per unit time as indicated by $T_{c 1}$ and $T_{c 2}$ parameters; it is quite rare to be outside of this interval since the $k_{c 1}$ and $k_{c 2}$ parameters are numerically rather large in absolute value which indicates a single entity behavior induced by the Gibbs probability density function whose single clique potentials are as those shown by Fig. 1a,

- quite likely to have intrinsic (self-generated) production, again 100-120 units per unit time, as indicated by $T_{p 1}$ and $T_{p 2}$ parameters; though it is still less likely to observe deviations outside of this interval, this is not as strict as the consumption, as implied by still strong $k_{p 1}$ and $k_{p 2}$ values which are weaker than $k_{c 1}$ and $k_{c 2}$,

- there is a strong coupling of both production and consumption levels between the directly connected two (left and right) neighbors; the levels of neighbors are unlikely to deviate more than 30 units, as indicated by $T_{v c}$ and $T_{v p}$ parameters. Since the likely production and consumption levels are both in the same [100120] interval, the system is balanced; in other words, the system does not have a preferred tendency to evolve towards systemic surplus or scarcity.

We ran a total of 20 simulations and recorded the number of surviving entities, at the steady-state, out of the initial 20 in each simulation. The term "steady-state" should be rather taken lightly: we run each simulator for a fixed amount of real-time that is long enough. These recorded number of surviving entities, and therefore, the number of defaults, may form the statistical basis for the assessment of the stability and the robustness of the network, as defined in Acemoglu et al. (2015).

To compare, we run another set of 20 simulations, by keeping all the parameters and the GRF Connectivity Matrix the same, but changing only the Debt Connectivity Matrix, so that an entity can grant/receive loans from any other entity in the network. Therefore, the statistical interactions form a ring type connected neighborhood network, whereas loan/debt interactions form a fully connected network. We observed that the average number of surviving entities are 12 for the ring type loan network, whereas, the average is 12.8 in the fully connected loan network. The estimated standard deviations are 1.3 and 1.6, respectively. Therefore, and as expected, we may conclude that a fully connected loan/debt network is slightly more robust compared to 
Table 2 Simulation 2.b parameters

\begin{tabular}{llllllllllllll}
\hline$k_{c 1}$ & $k_{c 2}$ & $T_{c 1}$ & $T_{c 2}$ & $k_{p 1}$ & $k_{p 2}$ & $T_{p 1}$ & $T_{p 2}$ & $v_{c 1}$ & $v_{c 2}$ & $T_{v c}$ & $v_{p 1}$ & $v_{p 2}$ & $T_{v p}$ \\
\hline-0.03 & 0.03 & 100 & 120 & -0.01 & 0.01 & 100 & 120 & -0.03 & 0.03 & 30 & -0.03 & 0.03 & 30 \\
\hline
\end{tabular}

the ring connected network, for these statistical interaction topology and parameters. However, we may also conclude that the steady-state statistics are not too different, and in both cases we do observe a kind of statistical convergence.

Simulation 2 In this simulation, we kept the parameters as in Table 1, but we changed the topology of both the GRF Connectivity Matrix and the Loan Connectivity Matrix to have full connectivity; therefore, each one of 20 entities are directly connected to all other 19 entities. Since the parameters that determine the strength of statistical coupling of production and consumption among the entities, $v_{c 1}, v_{c 2}, v_{p 1}$ and $v_{p 2}$, are kept the same, but the total number of neighborhood entity number is almost ten times increased (from 2 to 19), we expect two types of statistical results: (i) The average of 19 neighborhood consumption or production parameters will have a much smaller variance; however, the collective statistical influence will be tremendously stronger. In other words, for any entity, now it is almost improbable to deviate more than 30 units in amount of production or in amount of consumption, from the neighborhood induced rather stable average, at a given time. (ii) Even though the debt possibilities are now significantly relaxed, and therefore, it should be easier to cope with any financial crisis, the overall network of 20 entities are expected to collectively prosper, or collectively fall into extinction, as a consequence of implied strong coupling as a consequence of the associated parameters given in Table 1. Indeed, this is exactly what the simulation results show: In 11 of the 20 simulations we ran, we have seen an excellent survival, with good prosperity, of all 20 entities. But in 8 of those 20 simulations, the entire population defaulted (extinction). In one simulation, interestingly, it took longer to see a clear result, and 14 entities went to default, with rather short intervals between such defaults, but then the remaining healthy 6 of them surprisingly survived. We conclude from these observations that strong statistical coupling results in a strong convergence, but the state that is converged may be either one of the two extremes: overall prosperity or overall extinction. If accidentally, the overall system starts with a bright performance, the entire network will continue to prosper as a whole; on the other hand, if accidentally something goes wrong, the entire system collapses.

We then changed the interaction parameters of the model as given in Table 2.

The reductions in $k_{c 1}, k_{c 2}, k_{p 1}$ and $k_{p 2}$ indicate that the intrinsic (solo) production statistics are now more relaxed, and therefore, extreme variations on these components are allowed; the statistical behavior is now governed by a GRF probability density function whose single clique potentials are as in Fig. 1b. However, this is not the prime concern for the simulation we conducted. Rather, the reduction in coupling parameters, $v_{c 1}, v_{c 2}, v_{p 1}$ and $v_{p 2}$, brings the overall statistical influence of the neighbors (now we have 19 neighbors for each entity) pretty much to the level of overall influence of the two neighbors of Simulation 1. Please note that the debt structure is much more 
permissive since each entity can now grant or receive loans from all other entities. We see what we expect in this case: we saw that all 20 entities prosper, with zero default in any run. Due to ease in getting loans, and due to weaker coupling parameters that significantly reduces overall failures, the economic system operates perfectly well. No domino effect is observed.

Simulation 3 We also ran some experiments to test the system for negative impulsive inputs, using the parameters and the topology of the previous two examples. The simulator allows the user to pause the system at any instant, and change the storage value of any entity, externally. Using this facility, we ran the simulation for a while, and then paused it and manually change the storage value of the richest entity to zero, and then continued the iterations. Though we did not conduct extensive tests to draw conclusions, we observed that the external push to send one entity to default did not make a noticeable change in the statistical behavior of the network with the remaining entities. This feature is very useful to test the overall response of the system to external negative and positive impulsive inputs; it is possible to apply simultaneous changes to any number of entities at any time, and also, it is possible to repeat this as often as needed.

\section{Summary and Conclusions}

A complete statistical model for a collection of economic entities is given as a Gibbs random field. The statistical behavior of each entity, as well as the statistical behavior of the collection of those entities are modeled, and the model is complete in terms of its mathematical grounds. It is possible to look at the model in a hierarchical fashion by forming clusters of entities, if desired. Rather less complicated versions of the given model require a small number of parameters, and even in that case, still quite strong models, that are applicable to real-life situations, are feasible. It is possible to incorporate economic activities (consumption and production) as well as financial activities (loans) into the model. A computer simulator, based on state-ofthe-art technology is developed. The simulator has a friendly user interface, and gives the user a visual display to track the time evolution of the economic system, based on the presented model and user-chosen parameters. It is possible to track a large number of entities, where the number may go up to a few hundred thousands, both visually and through stored fine detail data. The simulator is based on a variation of the Metropolis algorithm. A few simulation results we have included in this paper show that it is possible to predict the statistical steady-state outcome of a given structure: The form and degree of statistical coupling among the entities, as well as the extent and topology of the loan neighborhood, may drastically influence the overall behavior, as expected. As a consequence of the provided model and the simulator, now it is possible to test different forms and degrees of couplings among the entities, and different forms of financial (loan/debt) network topologies. The results may be useful for the community who conducts research in economic and financial policies and their expected outcomes.

In line with the goals of the paper that are outlined in Section 1 (i) a general, flexible and hierarchical random field model is presented for economic and financial activities; (ii) the model can represent many different situations where simple local entities, which 
directly interact only with their neighbors, form large size aggregates; (iii) a simulator, with a user friendly interface and a full user's manual, is written and implemented, (iv) a simple set of simulations to check the resultant global effects of simple entity behavior in an hypothetical financial network is run and interesting results are obtained; (v) even such simple simulations show that the developed tool can be used to test robustness of those hypothetical financial networks; (vi) the developed simulation tool can be used to track and test the time evolution, and eventually the steady-state behavior, of a large size network; (vii) the developed model and the associated simulator may be used to test the resultant macro effects of various local economic behavior and interactions; (viii) the presented tools may be used to develop and test policies that target micro level behavior changes to achieve desired global effects; (ix) the presented tools may be used to check some theoretical results presented in the literature via designed simulations that generate the same network topologies and use the same parameters.

We have already got some interesting results as a consequence of the simulations presented in this paper. For example, we observed that a fully connected financial network, that represents the loan activities among interacting entities which also produce and consume, is more robust compared to a sparse ring-connected financial network; however, the difference is rather small if the underlying direct influences among the consuming and producing entities are restricted to a ring-connected topology. If, on the other hand, the underlying network is also fully connected, where the production and consumption behavior of each entity is affected by all other entities, the parallel running fully connected debt/loan network is significantly more robust, compared to its ring-connected counterpart; however, even in such a case, too strong economic behavior couplings among entities may not be desirable since we observed occasional default (extinction) of the entire population due to prevailing domino effect; instead, if the couplings are somewhat weakened, we always observed total prosperity. These observations imply that balanced aggregate consumption and production levels in a society, together with well-connected but not too strong economic behavioral influences and well-extended loan/debt interactions among the entities lead to total prosperity of all entities.

In conclusion we described a simulation tool that can be utilized in several manners for policy analysis and research: e.g., it can be used to check computationally the theoretical results obtained, for example, by Acemoglu et al. (2015) for network contagion in case of financial shocks, and to extend the results to cases which are not amenable to theoretical analysis due to increased complexity. Or it may mimic an actual interbank/financial network with all its connections after a careful calibration to relevant data to observe the impact of different scenarios to cthe current financial system. The possibilities for future uses are indeed numerous.

The presented work does not attempt to estimate, or adapt, the parameters of the presented GRF model to fit the model to a set of real-life data. However, this is a feasible future work.

Furthermore, the model is quite flexible to handle heterogeneous networks, as well as time-varying trends; we presented the general model which can easily handle such cases, but we did not included those in the current simulator. This could also be a future work. 
As the topic of the present paper entails inferences about aggregate macroeconomic variables departing from microeconomic behavior it would be natural to study models on the links between microecomic entities to macroeconomic level. A recent study by Acemoglu et al. (2017) argues that aggregating microeconomic variables under a Gaussian distribution assumption and using the central limit theorem may fail to explain macroeconomic tail risks. They use a model economy of $n$ competitive sectors that are linked to each other and are subject to idiosyncratic productivity shocks. Their results indicate that some idiosyncratic microeconomic shocks can be at the origin of such macroeconomic downturns. The paper contains an extensive list of references on related literature. A simulation-based evaluation of the model of Acemoglu et al. (2017) could be undertaken as future study using the software tools of the present paper.

Obviously, extensive sets of simulations must be conducted to come up with dependable empirical results. But an alternative is to mathematically investigate the underlying Markov chains and, if possible, to come up with theoretical results on the transient and steady-state behavior, at least for some cases. Neither of these were the purposes of this paper; we have just provided a mathematical tool, together with a simulator, to pave the way for such research work.

Acknowledgements The simulator software is developed and written by Can Furtına. A very preliminary version of the simulator was written by Çă̆atay Günay.

\section{References}

Acemoglu, D., Ozdaglar, A., \& Tahbaz-Salehi, A. (2015). Systemic risk and stability in financial networks. American Economic Review, 105(2), 564-608. https://doi.org/10.1257/aer.20130456.

Acemoglu, D., Ozdaglar, A., \& Tahbaz-Salehi, A. (2017). Microeconomic origins of macroeconomic tail risks. American Economic Review, 107(1), 54-108. https://doi.org/10.1257/aer.20151086.

Afonso, G., Kovner, A., \& Schoar, A. (2011). Stressed, not frozen: The federal funds market in the financial crisis. The Journal of Finance, 66(4), 1109-1139. https://doi.org/10.1111/j.1540-6261.2011.01670. $\mathrm{X}$.

Afonso, G., \& Lagos, R. (2015). Trade dynamics in the market for federal funds. Econometrica, 83(1), 263-313. https://doi.org/10.3982/ECTA10586.

Amini, H., Cont, R., \& Minca, A. (2016). Resilience to contagion in financial networks. Mathematical Finance, 26(2), 329-365. https://doi.org/10.1111/mafi.12051.

Bargigli, L., di Iasio, G., Infante, L., Lillo, F., \& Pierobon, F. (2015). The multiplex structure of interbank networks. Quantitative Finance, 15(4), 673-691.

Bech, M. L., \& Atalay, E. (2010). The topology of the federal funds market. Physica A: Statistical Mechanics and its Applications, 389(22), 5223-5246.

Beichl, I., \& Sullivan, F. (2000). The Metropolis algorithm. Computing in Science Engineering, 2(1), 65-69.

Besag, J. (1974). Spatial interaction and the statistical analysis of lattice systems. Journal of the Royal Statistical Society Series B (Methodological), 36(2), 192-236.

Derin, H., \& Kelly, P. A. (1989). Discrete-index markov-type random processes. Proceedings of the IEEE, 77(10), 1485-1510.

Diebold, F. X., \& Y1lmaz, K. (2014). On the network topology of variance decompositions: Measuring the connectedness of financial firms. Journal of Econometrics, 182(1), 119-134, causality, prediction, and specification analysis: Recent advances and future directions.

Firtına, C. (2015). User's manual for the GMRF economic activity simulator.

Fuhrer, J. C. (2000). Habit formation in consumption and its implications for monetary-policy models. American Economic Review, 90(3), 367-390. https://doi.org/10.1257/aer.90.3.367. 
Glasserman, P., \& Young, H. P. (2016). Contagion in financial networks. Journal of Economic Literature, 54(3), 779-831. https://doi.org/10.1257/jel.20151228.

Gofman, M. (2012). A network-based analysis of over-the-counter markets. In AFA 2012 Chicago meetings paper.

Gofman, M. (2017). Efficiency and stability of a financial architecture with too-interconnected-to-fail institutions. Journal of Financial Economics, 124(1), 113-146.

Gurelli, M. I., \& Onural, L. (1994). On a parameter estimation method for Gibbs-Markov random fields. IEEE Transactions on Pattern Analysis and Machine Intelligence, 16(4), 424-430.

Hammersley, J. M., \& Clifford, P. (1971). Markov random fields on finite lattices and graphs. Unpublished.

Havranek, T., Rusnak, M., \& Sokolova, A. (2017). Habit formation in consumption: A meta-analysis. European Economic Review, 95, 142-167.

Haykin, S. (1986). Adaptive filter theory. Prentice-Hall information and system sciences series. Upper Saddle River: Prentice-Hall.

Isham, V. (1981). An introduction to spatial point processes and Markov random fields. International Statistical Review/Revue Internationale de Statistique, 49(1), 21-43.

Leitner, Y. (2005). Financial networks: Contagion, commitment, and private sector bailouts. The Journal of Finance, 60(6), 2925-2953. https://doi.org/10.1111/j.1540-6261.2005.00821.x.

Lucas, J. R. E. (1976). Economic policy evaluation: A critique. In K. Brunner \& A. H. Meltzer (Eds.), The Philips curve and labor markets, Carnegie-Rochester conferences on public policy (pp. 19-46). Amsterdam: North Holland.

Malamud, S., \& Rostek, M. (2017). Decentralized exchange. American Economic Review, 107(11), 332062. https://doi.org/10.1257/aer.20140759.

Nelson, R., Dosi, G., Helfat, C., Pyka, A., Saviotti, P. P., Lee, K., et al. (2018). Modern evolutionary economics: An overview. Cambridge: Cambridge University Press.

Onural, L. (1988). Vector Gibbs random fields and colored textures. In E. Gelenbe, E. Orhun, \& E. Başar (Eds.), The Third international symposium on computer and information sciences (ISCIS), Izmir, Turkey.

Onural, L. (2016). Gibbs random fields and Markov random fields with constraints.

Onural, L. (1991). Generating connected textured fractal patterns using markov random fields. IEEE Transactions on Pattern Analysis and Machine Intelligence, 13(8), 819-825.

Peltonen, T. A., Scheicher, M., \& Vuillemey, G. (2014). The network structure of the CDS market and its determinants. Journal of Financial Stability, 13, 118-133.

Spitzer, F. (1971). Markov random fields and Gibbs ensembles. The American Mathematical Monthly, $78(2), 142-154$.

Zawadowski, A. (2013). Entangled financial systems. The Review of Financial Studies, 26(5), 1291-1323. https://doi.org/10.1093/rfs/hht008.

Publisher's Note Springer Nature remains neutral with regard to jurisdictional claims in published maps and institutional affiliations. 